\title{
ON THE DEVELOPMENT OF THE BRASS-TYPE TEXTURE IN AUSTENITIC STAINLESS STEEL*
}

\author{
C. D. SINGH \\ Research and Development Centre for Iron and Steel, Steel Authority \\ of India Limited, Ranchi - 834 002, India
}

It has been clarified and demonstrated that the conclusions drawn by Singh, Ramaswamy and Suryanarayana (1992) in an investigation of development of rolling textures in an austenitic stainless steel are correct. The observations and reinterpretations drawn by Leffers (1993) are without any proper scientific basis and do not hold good at least in austenitic stainless steel.

KEY WORDS: Rolling texture, copper-type, brass-type, mechanical twinning.

\section{INTRODUCTION}

FCC materials possess different rolling textures i.e. the copper-type and the brass-type and these textures are described in terms of two limited fibres (i.e. a fibre axis $\langle 110\rangle / / \mathrm{ND}$ extending from $\{011\}\langle 100\rangle$ to $\{011\}\langle 112\rangle$ and the other fibre $\langle 110\rangle 60^{\circ} \mathrm{ND}$ running from $\sim\{112\}\langle 111\rangle$ through $\sim\{123\}\langle 634\rangle$ to $\{011\}\langle 112\rangle$ with $\langle 110\rangle$ axis inclined about $60^{\circ}$ from ND towards RD). While the brass-type texture in low stacking fault energy (SFE) materials is characterised by $\langle 110\rangle / / \mathrm{ND}$ fibre with $\{011\}\langle 112\rangle$ as a major component, the copper-type (high and medium SFE materials) is described mainly by $\langle 110\rangle 60^{\circ} \mathrm{ND}$ fibre with $\{112\}\langle 111\rangle$ or $\{123\}\langle 634\rangle$ as major orientations. It is well established and documented in the literatures (Hirsch and Lücke, 1988a; Hirsch, Lücke and Hatherly, 1988b) that the textures at low degrees of rolling (i.e. 20\% reduction) are identical in all FCC materials i.e. high SFE (A1), medium SFE(Cu) and low $\mathrm{SFE}(\alpha$-brasses) and consist of orientations concentrated homogeneously along two fibres $\left(\langle 110\rangle / / \mathrm{ND}\right.$ and $\left.\langle 110\rangle 60^{\circ} \mathrm{ND}\right)$. At medium degrees of rolling (i.e. $\sim 60 \%$ reduction) only the $\langle 110\rangle 60^{\circ} \mathrm{ND}$ fibre for high and medium SFE materials and the $\langle 110\rangle / \mathrm{ND}$ fibre for low SFE materials remain. The $\langle 110\rangle 60^{\circ} \mathrm{ND}$ fibre deteriorates to one or more peaks in $\{112\}\langle 111\rangle /\left\{\begin{array}{ll}4 & 41\end{array}\right\}\langle 11118\rangle,\{123\}\langle 634\rangle$ and $\{011\}\langle 112\rangle$ and the $\langle 110\rangle / / N D$ fibre degenerates to only $\{011\}\langle 112\rangle$ with very little $\{011\}\langle 100\rangle$ at high degree of rolling (i.e. 95\% reduction). The structure of these experimentally observed rolling textures (both early and final stages) for high and medium SFE materials agrees fairly well with that of the simulated textures calculated on the basis of Taylor-type models [i.e. (FC) Full Constraints (Taylor, 1938) and Relaxed Constraints (RC)] assuming multiple slip during rolling deformation (Hirsch and Lücke, 1988c).

\footnotetext{
- This paper is a reply to the comment by T. Leffers, pp. 53-58 of this issue of Textures \& Microstructures.
} 
For low SFE materials ( $\alpha$-brass) after initial development of $\langle 110\rangle / / \mathrm{ND}$ and $\langle 110\rangle 60^{\circ} \mathrm{ND}$ fibres, the texture transition from copper-type to brass-type has been found to occur according to Wassermann's mechanism (Wassermann, 1963) where mechanical twinning along with deformation inhomogeneities play a dominant role in the formation of final brass-type texture (Hirsch, Lücke and Hatherly, 1988b). Here, mechanical twinning transforms the orientations in the vicinity of $\{112\}\langle 111\rangle$ to orientations near $\{552\}\langle 115\rangle$ and also twins the orientations near $\{123\}\langle 634\rangle$ to its own symmetrical positions and thus causes an abrupt decrease in the orientation density of $\{112\}\langle 111\rangle$ and also simultaneous increase in the density of the twin component $\{552\}\langle 115\rangle$. This decrease in the density of $\{112\}\langle 111\rangle$ along with simultaneous increase in $\{552\}\langle 115\rangle$ marks the beginning of texture transition and occurs at critical degree of rolling which decreases with decreasing SFE. In case of low $\mathrm{Zn}$ brass, further rolling causes normal slip rotation of $\{552\}\langle 115\rangle$ towards $\{011\}\langle 100\rangle$ and then to $\{011\}\langle 112\rangle$ and finally leads to the formation of brass-type texture, i.e. $\langle 110\rangle / / \mathrm{ND}$ fibre. On the other hand, for high $\mathrm{Zn}$ brass, the abnormal slip rotation of twin $\{552\}\langle 115\rangle$ - parent $\{112\}\langle 111\rangle$ lamellae towards i.e. $\{111\}\langle 112\rangle$ followed by the formation of non-crystallographic shear band formation and subsequently the resumption of normal slip in the fine grains of $\{011\}\langle 100\rangle$ towards $\{011\}\langle 112\rangle$ lead to the formation of brass-type texture (Duggan et al., 1978). Recently, Singh, Ramaswamy and Suryanarayana (1992), have supported the view point of Duggan et al. (1978) on the basis of the statistical appearance of the structure of ODF obtained in an austenitic stainless steel cold rolled at $473 \mathrm{~K}$ and also using the existing informations on microscopical observations of deformation inhomogeneities available in the literatures (Blicharski and Gorczyca, 1978; Hutchinson, Duggan and Hatherly, 1979).

On the contrary, Leffers and coworkers (Leffers and Grum Jensen, 1968; Leffers, 1969; Pedersen and Leffers, 1987; Leffers and Juul Jensen, 1988; Leffers and BlideSorensen, 1990; Leffers and Juul Jensen, 1991; Leffers and Hansen, 1992) have disputed and rejected Wassermann's twinning hypothesis and also suggested that the texture transition from copper-type to brass-type can be accounted for by using classical lowerbound Sachs model (Sachs, 1928) or modified Sachs model. Furthermore, they also claim that from the very beginning of rolling deformation, the development of textures in low SFE materials is totaly different from that in high and medium SFE materials. In their simulation, they assume the occurrence of single slip in all grains. This arguments has been negated by a considerable body of research works. It is worth to mention that the Sachs type models do not simulate the formation and simultaneous destruction of the texture component near $\{111\}\langle 112\rangle$ which occurs as a result of mechanical twinning and abnormal slip rotation on one hand and the shear bands formation on the other. Once again, Leffers (1993) has doubted the validity of Wassermann's twinning concept as a mechanism responsible for the transition of texture from copper-type to brass-type during early stage of deformation (upto about $50 \%$ cold reduction) and also tried to reinterpret the results obtained by Singh, Ramaswamy and Suryanarayana (1992) in terms of the above mentioned suggestions.

The aim of the present work is to clarify and to demonstrate that the statements [i.e. a) the initial development of brass-type texture is similar to that of copper-type and b) this development follows the FC Taylor model - at least upto $30 \%$ cold reduction - and finally c) mechanical twinning has direct effect on the texture transition during further rolling ( $\leq 50 \% C R]$ made by Singh, Ramaswamy and Suryanarayana (1992) are correct and also that Leffers's reinterpretations do not hold good in this case. These clarifications are based on development of textures and on the evolution of 
microstructures as determined by orientation distribution functions (ODF) technique and transmission electron-microscopy (TEM) respectively. The details of the ODF technique used to analyse the textures have been given in the previous work (Singh, Ramaswamy and Suryanarayana (1992) whereas TEM has been used to examine the thin foils parallel to rolling plane and to provide further evidences on microstructural evolution during rolling.

\section{TEXTURE DEVELOPMENT}

The hot band (HB) of austenitic stainless steel possesses a sharp starting texture and has been characterised by the appearance of strong components at the position of the orientations $\{011\}\langle 112\rangle, \sim\{123\}\langle 634\rangle$ and $\{001\}\langle 100\rangle$ as well as of minor components at $\{225\}\langle 554\rangle$ and also along RD rotated cubes i.e. $\{013\}-\{012\}-\{011\}\langle 100\rangle$ (Singh, Ramaswamy and Suryanarayana, 1991). The orientations $\{225\}\langle 554\rangle, \sim\{123\}\langle 634\rangle$ and $\{011\}\langle 112\rangle$ are the essential components of rolling texture (i.e. $\langle 110\rangle 60^{\circ} \mathrm{ND}$ fibre) while the component $\{001\}\langle 100\rangle$ along with other RD rotated cubes constitute the recrystallisation texture. As an extraction of the work (Singh, Ramaswamy and Suryanarayana, 1992) Figure 1 shows a series of $\emptyset_{2}=45^{\circ}$ sections of ODF for different degrees of rolling. Most of the important orientations except $\sim\{123\}\langle 634\rangle$ are contained
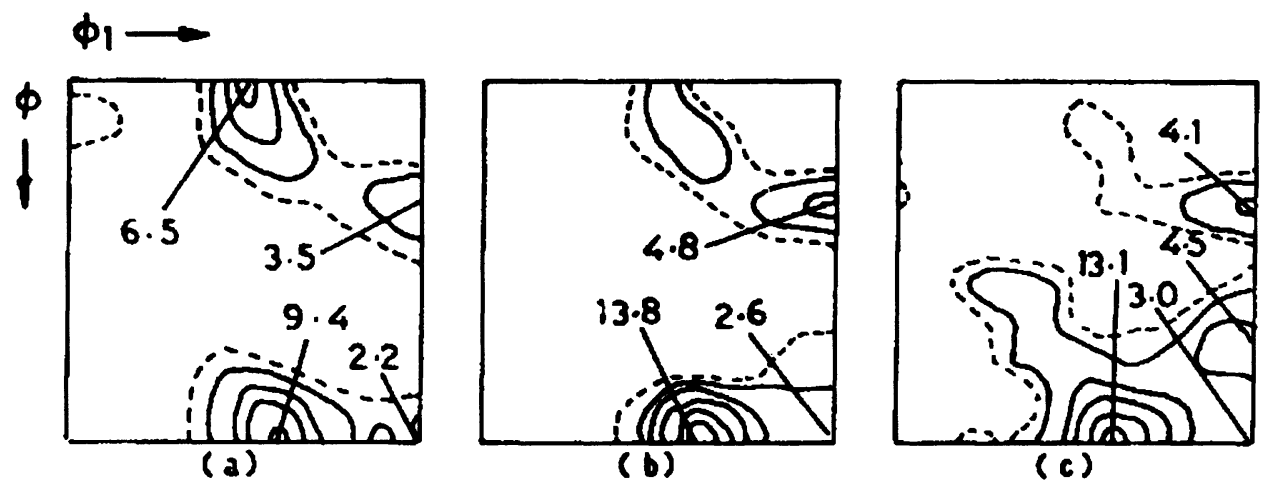

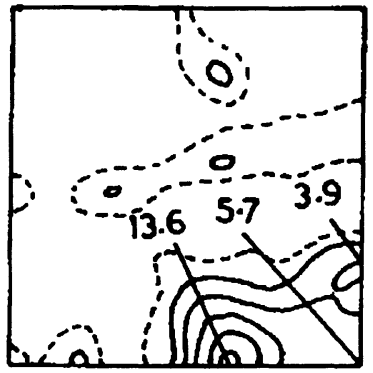

(d)

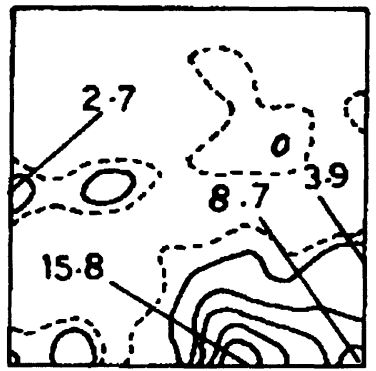

(e)

Figure $1 \emptyset_{2}=45^{\circ}$ sections of the ODF for different cold reduction(CR) : a) 0\%CR; levels-1,2,4,6,9 b) $30 \% \mathrm{CR}$; levels-1,2,4,6,9,12 c) $50 \% \mathrm{CR}$; levels-1,2,4,6,9,12 d) $70 \% \mathrm{CR}$; levels-1,2,4,7,10,13 d) $90 \% \mathrm{CR}$; levels- $1,2,5,8,11,14$. 
in this section. In addition, this section also shows the position and density of many orientation fibres such as $\langle 110\rangle / / \mathrm{ND},\langle 110\rangle / / \mathrm{TD}$ and $\langle 111\rangle / / \mathrm{ND}$. This figure clearly reveals the depletion of cube orientation $\{001\}\langle 100\rangle$ with increasing deformation and finally this orientation disappears at $50 \%$ reduction. Simultaneously with the decrease in the density of $\{001\}\langle 100\rangle$, there is increase in the orientation density of the components $\{225\}\langle 554\rangle$ and $\sim\{123\}\langle 634\rangle$.

The distribution of orientation density along $\langle 110\rangle / / \mathrm{ND}$ fibre and $\langle 110\rangle 60^{\circ} \mathrm{ND}$ fibre are shown in Figures 2 and 3 respectively. It can be inferred from these figures that at low degrees of rolling ( $\leq 30 \% \mathrm{CR}$ ) the density of all texture components of two fibres (i.e. $\langle 110\rangle / / \mathrm{ND}$ and $\langle 110\rangle 60^{\circ} \mathrm{ND}$ ) increases in general and in particular this increase is more at $\{011\}\langle 112\rangle$. At medium degrees of rolling $(\leq 50 \% \mathrm{CR})$, practically no major

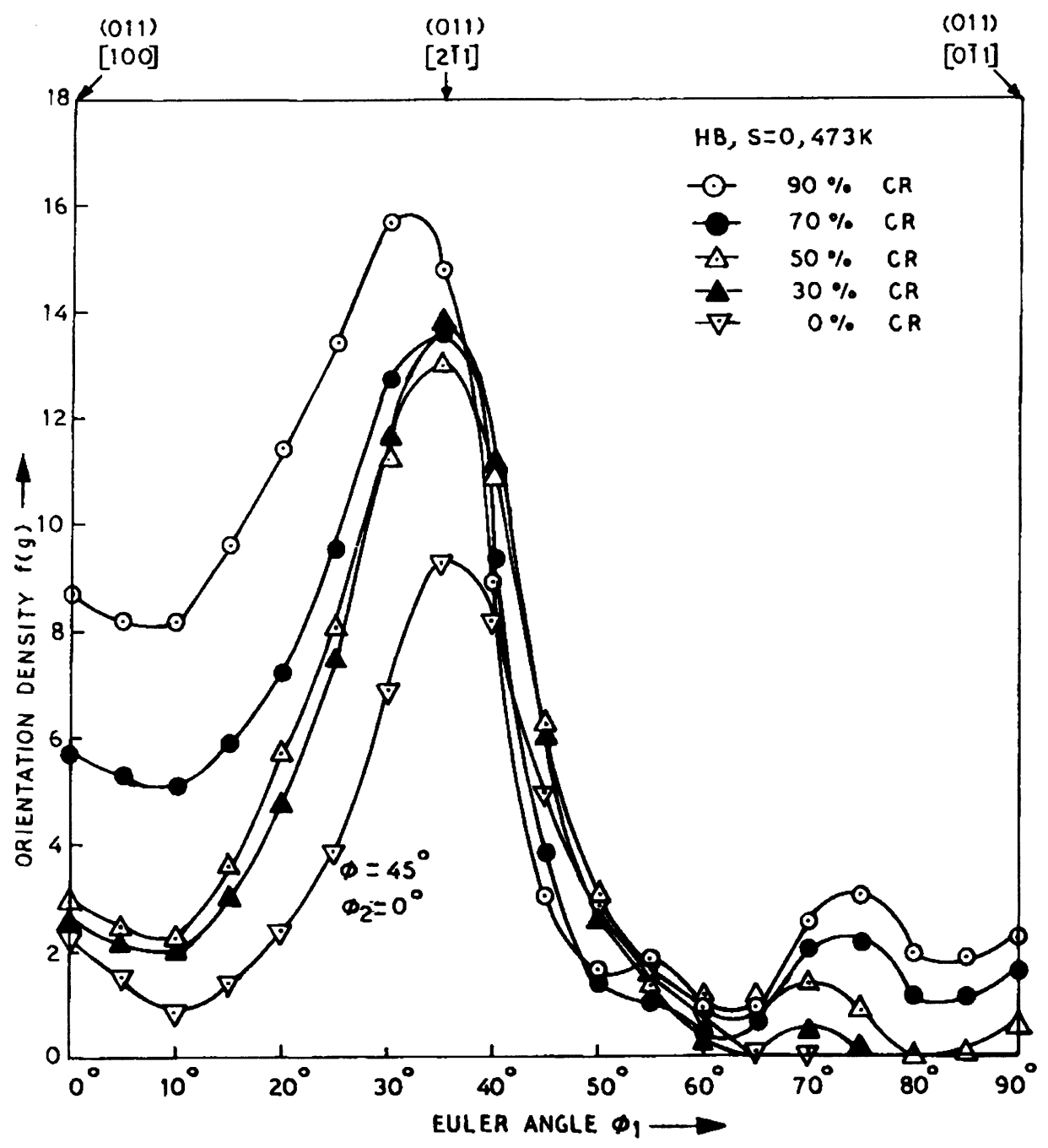

Figure 2 Orientation density along $\langle 110\rangle / / \mathrm{ND}$ fibre for different cold reduction (CR). 


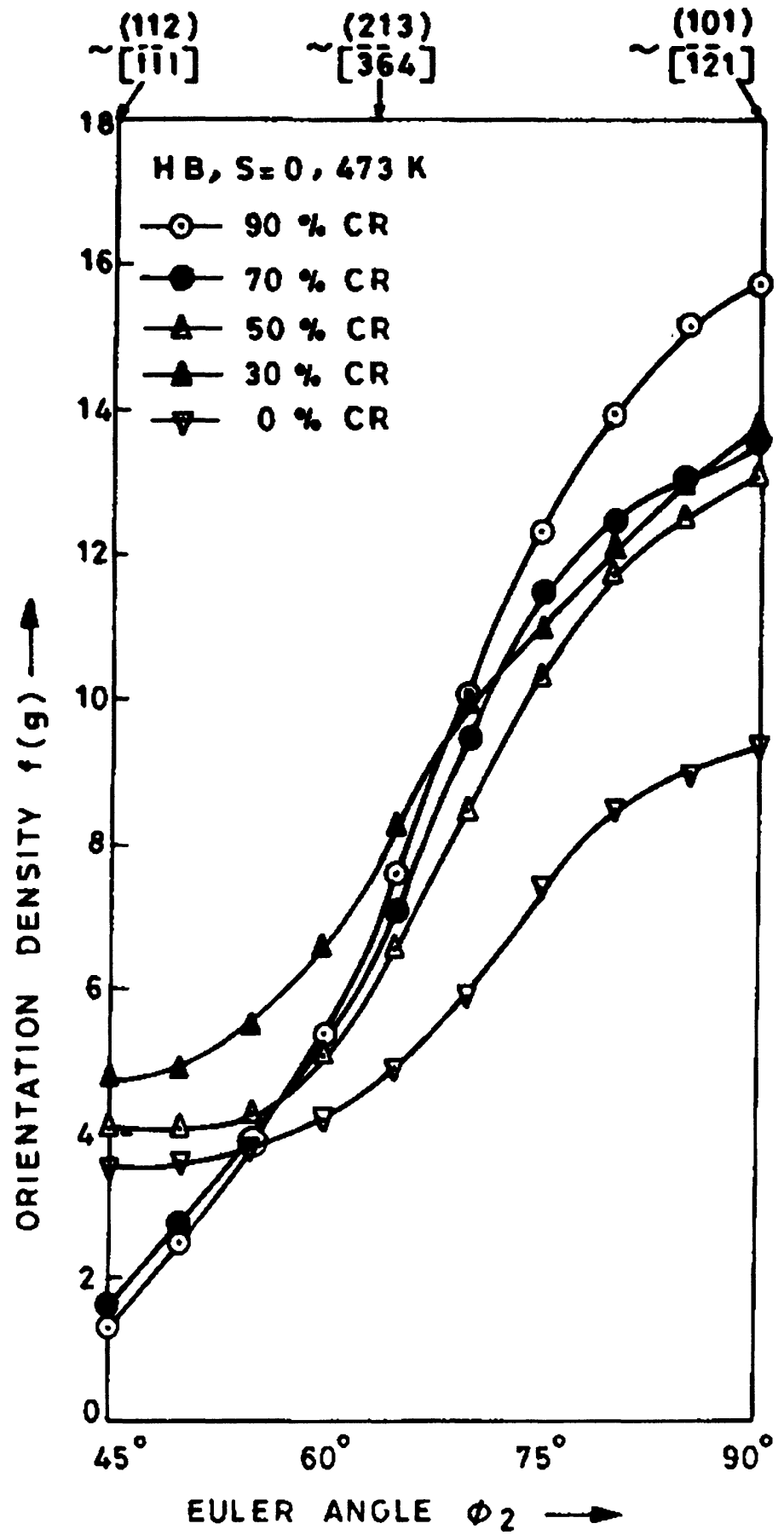

Figure 3 Orientation density along $\langle 110\rangle 60^{\circ}$ ND fibre for different cold reduction (CR). 
changes in orientation density of $\langle 110\rangle / / \mathrm{ND}$ fibre have been observed, whereas the densities of the components $\{225\}\langle 554\rangle$ and $\sim\{123\}\langle 634\rangle$ of $\langle 110\rangle 60^{\circ} \mathrm{ND}$ fibre have started decreasing. However, there is very slight increase in the density of $\{011\}\langle 100\rangle$.

Figure 4 reveals clearly the plot of orientation density of all components with a common axis $\langle 110\rangle / / \mathrm{TD}$ fibre against $\emptyset$ at $\emptyset_{1}=90^{\circ}, \emptyset_{2}=45^{\circ}$. The $\langle 110\rangle / / \mathrm{TD}$ fibre axis includes the component $\{225\}\langle 554\rangle$ at $\varnothing=30^{\circ}$, its twin $\{144\}\langle 811\rangle$ at $\varnothing=80^{\circ}$, the component $\{011\}\langle 100\rangle$ at $\emptyset=90^{\circ}$ and the component $\{111\}\langle 112\rangle$ which is an intermediate orientation at $\emptyset=55^{\circ}$. At low degrees of rolling (i.e. at $30 \% \mathrm{CR}$ ) one can easily notice a clear peak at $\{225\}\langle 554\rangle$ and a scattering of $\{011\}\langle 100\rangle$ towards $\{144\}\langle 811\rangle$ at $\emptyset=80^{\circ}$

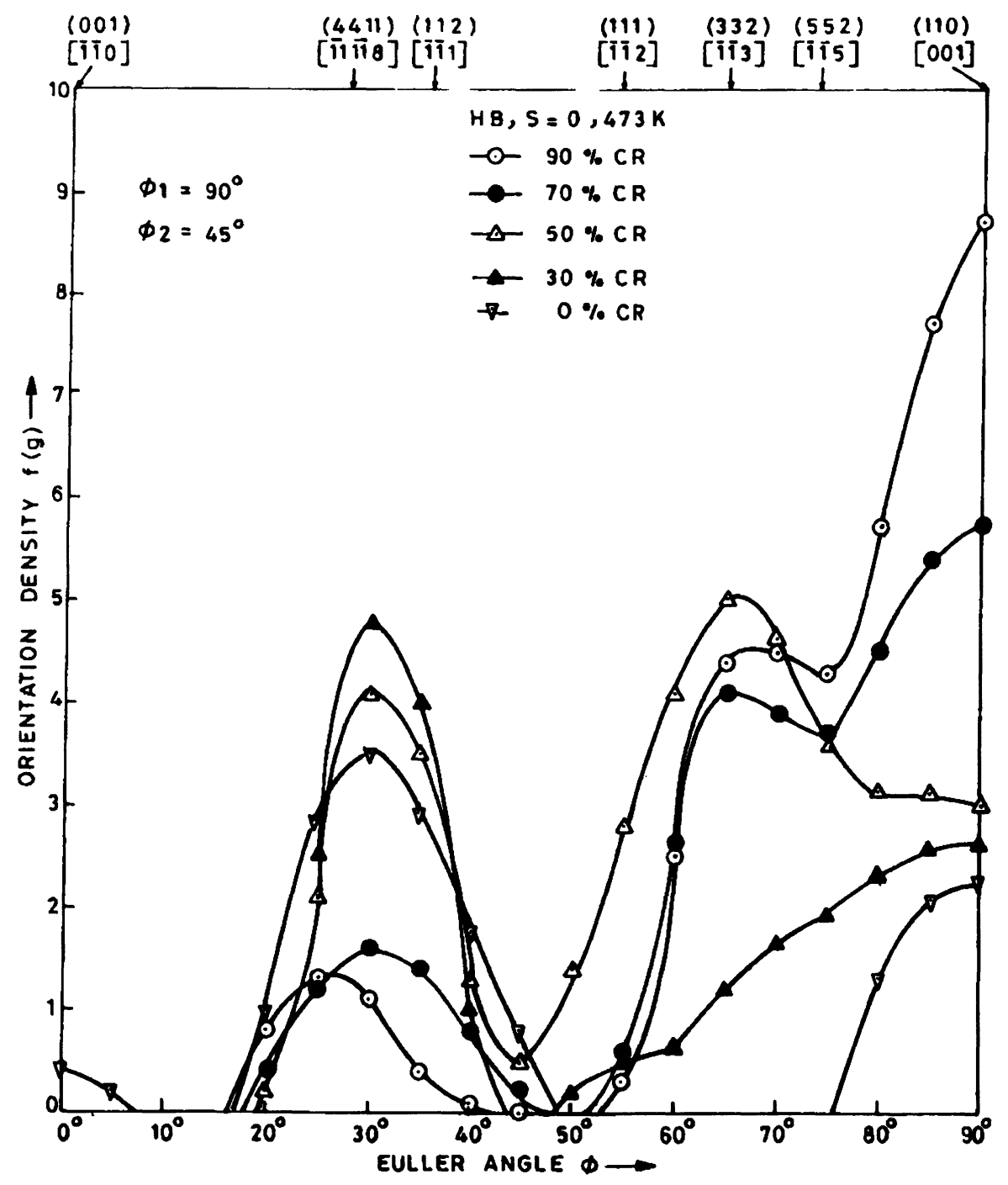

Figure 4 Orientation density along $\langle 110\rangle / / T \mathrm{TD}$ fibre for different cold reduction (CR). 


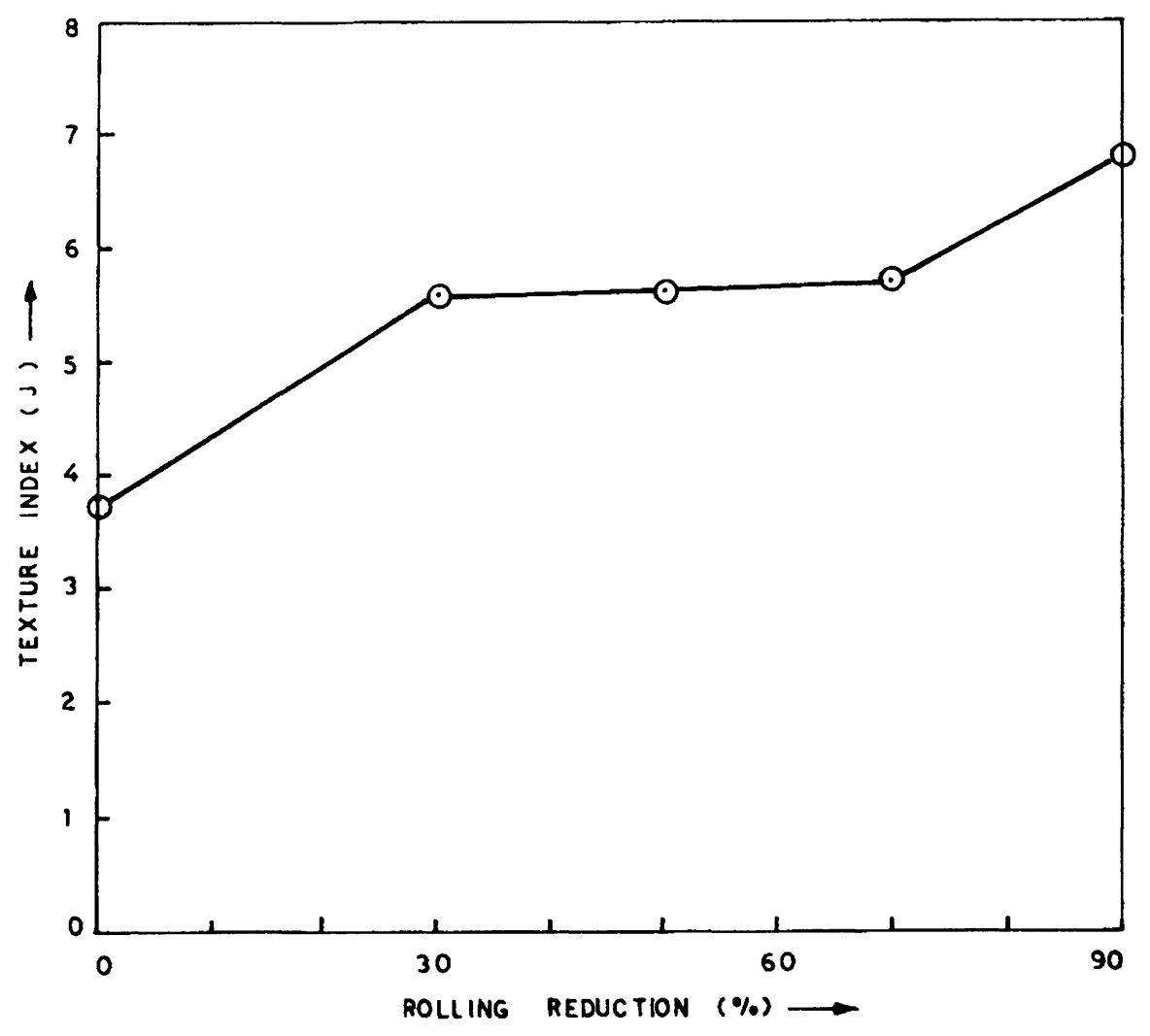

Figure 5 Plot of texture index with rolling reduction.

and also towards $\{332\}\langle 113\rangle$ at $\emptyset=65^{\circ}$. For further rolling ( $\left.\leq 50 \% \mathrm{CR}\right)$ there is reduction in density of $\{225\}\langle 554\rangle$ and simultaneously an increase in the density of $\{332\}\langle 113\rangle$ and $\{144\}\langle 811\rangle$.

On the other hand, Figure 5 shows the plot of average sharpness of texture (texture index $\mathrm{J}$ ) with increasing degrees of rolling. This figure indicates a monotonous increase in the texture index upto $30 \% \mathrm{CR}$ and then it remains constant for further rolling (i.e. $>30 \% \mathrm{CR}$ and $\leq 70 \% \mathrm{CR}$ ) and finally it again increases for higher degrees of rolling $(>70 \% \mathrm{CR})$. The exact position of the $\langle 110\rangle 60^{\circ} \mathrm{ND}$ fibres in Euler space are given in the form of their coordinates $\emptyset_{1}$ and $\varnothing$ and plotted as a function $\emptyset_{2}$ (Figure 6) for various degrees of rolling (i.e. $0 \% \mathrm{CR}, 30 \% \mathrm{CR}$ and $50 \% \mathrm{CR}$ ). Furthermore, the extracted data of the position of these fibres (Hirsch and Lücke, 1988c) calculated on the basis of FC Taylor model based on the assumption of homogeneous multiple slip for various degrees of rolling ( $18 \% \mathrm{CR}, 33 \% \mathrm{CR}$ and $45 \% \mathrm{CR}$ ) have been superimposed on Figure 6. It is interesting to note that the experimentally observed $\langle 110\rangle 60^{\circ} \mathrm{ND}$ fibre for $\mathrm{HB}(0 \% \mathrm{CR})$ runs very close to the $\emptyset$ and $\emptyset_{1}$ lines of theoretically determined $\langle 110\rangle 60^{\circ} \mathrm{ND}$ fibre calculated on the basis of FC Taylor model. The orientations of these experimental $\langle 110\rangle 60^{\circ} \mathrm{ND}$ fibres are systemetically shifted towards higher $\emptyset$ and $\emptyset_{1}$ values in the vicinity of the orientation $\{011\}\langle 112\rangle$. With increase in the degrees of rolling, these 


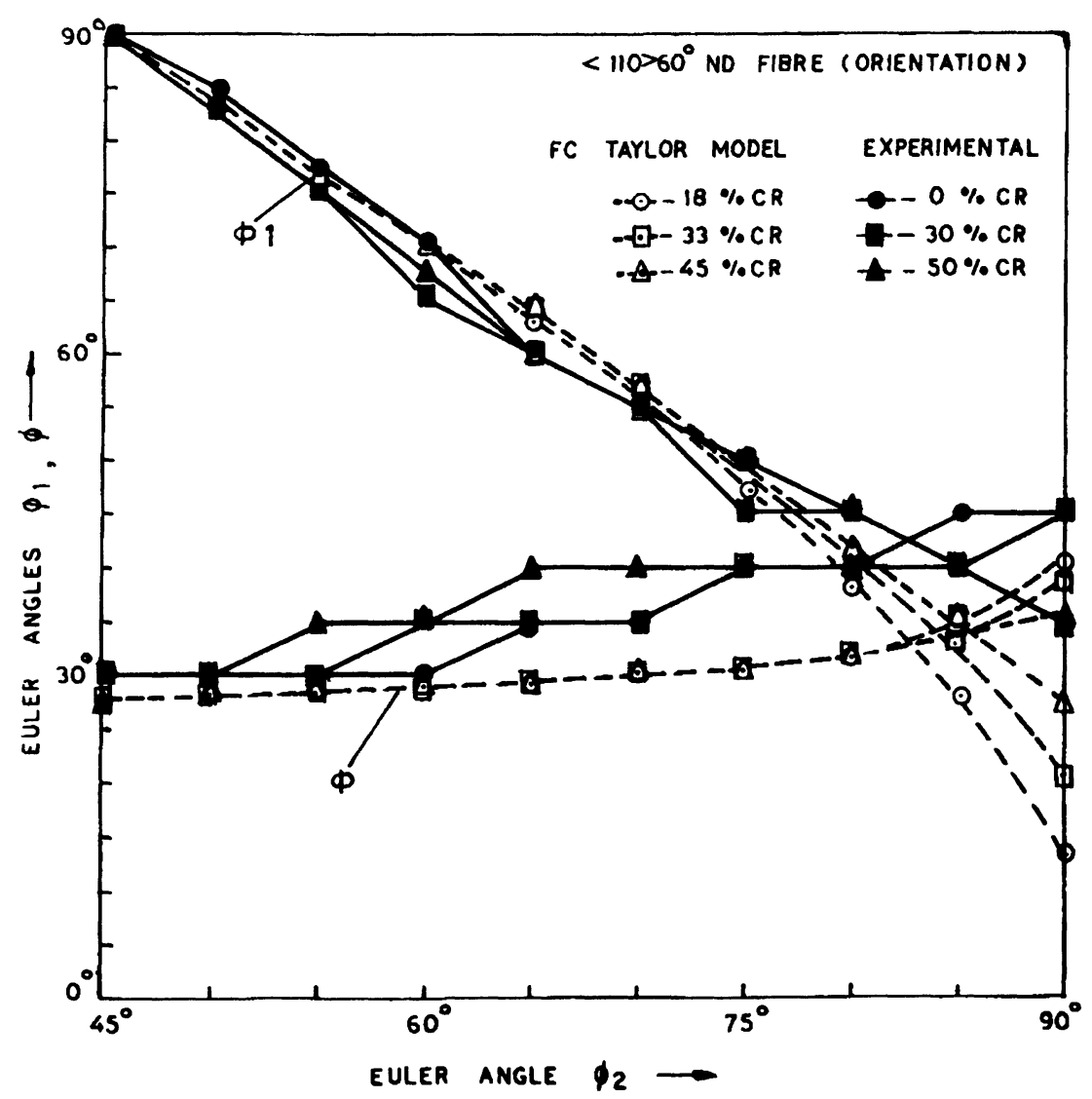

Figure 6 Orientation of $\langle 110\rangle 60^{\circ} \mathrm{ND}$ fibre in the Euler space given by $\emptyset_{1}, \varnothing$ as function of $\emptyset_{2}$.

fibres further shift towards higher $\emptyset$ and lower $\emptyset_{1}$ values in the region of orientations between $\sim\{123\}\langle 634\rangle$ and $\{225\}\langle 554\rangle$. This progressive shift towards higher $\emptyset$ values occurs in the deformation range where mechanical twinning predominates.

\section{MICROSTRUCTURE EVOLUTION}

The initial structure of HB has been described by the presence of mostly elongated grains as well as of some recrystallised grains (Singh, Ramaswamy and Suryanarayana, 1991). Furthermore, this initial structure also exhibits a full grown recovery twin along with a number of stacking faults within a grain [Figure 7(a)], while selected area diffraction (SAD) pattern of the faulted region is shown in Figure 7(b). The indexing of the SAD pattern is given in Figure 7(c). In Figure 7 the habit plane of the faults $\{111\} \gamma$ is oriented parallel to the $\langle 110\rangle \gamma$ electron beam direction and from the relative rotation of the bright field (BF) and SAD pattern the faults are seen to lie along $\langle 112\rangle$ direction. The extent of the intensity streaking in the SAD pattern is indicative of extremely thin faults. The SAD pattern taken from the region containing recovery twin 

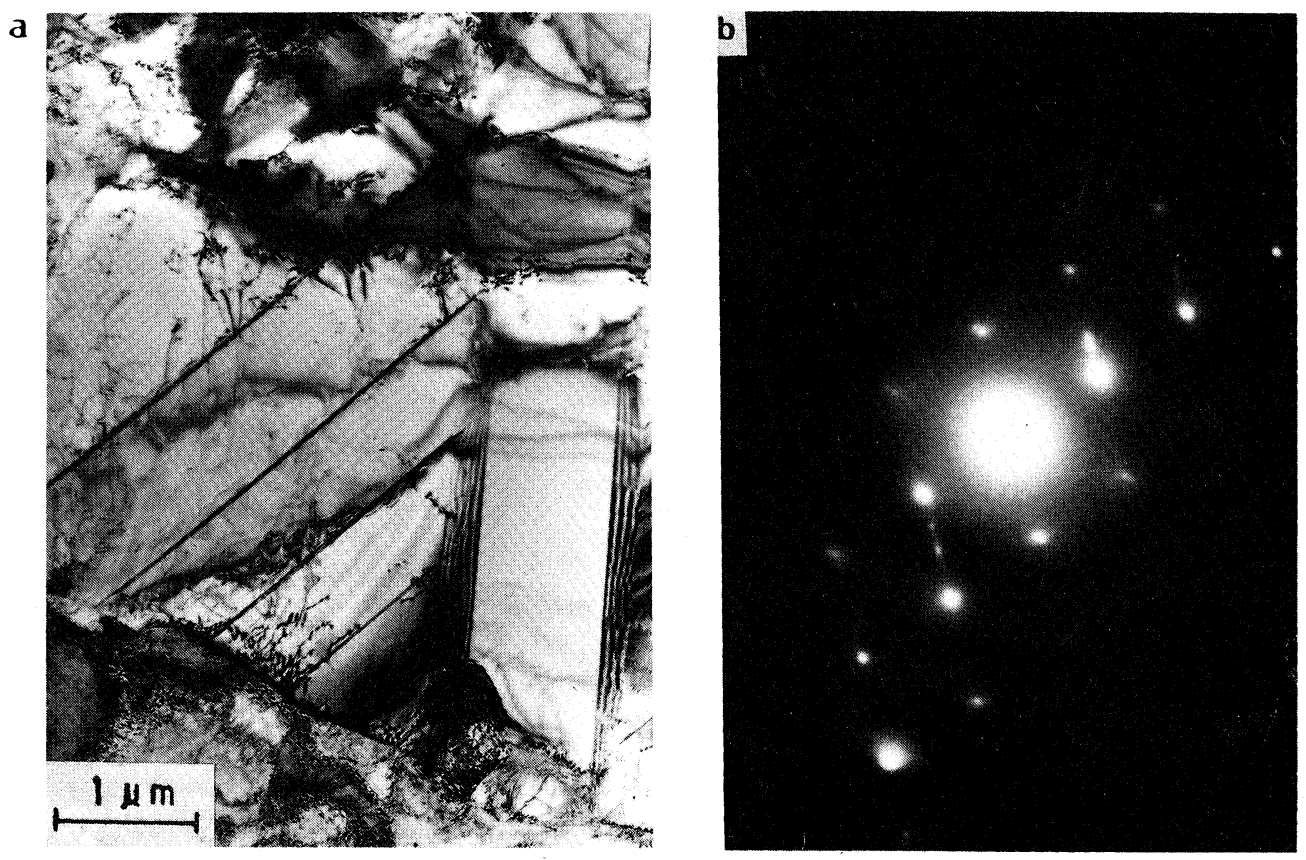

c

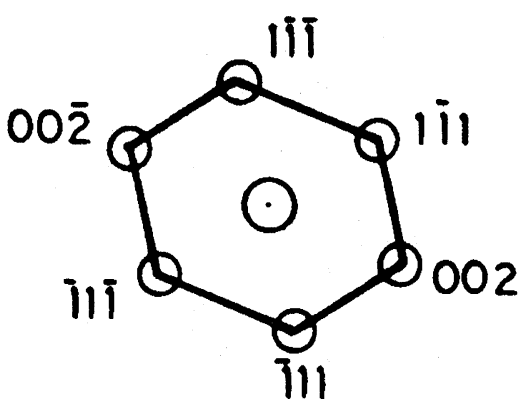

\section{$\left[\begin{array}{lll}1 & 0\end{array}\right]_{Y}$}

Figure 7 Transmission electron micrographs of $\mathrm{HB}(0 \% \mathrm{CR})$ : a) bright field (BF) image b) selected area diffraction (SAD) pattern c) key to SAD pattern. 


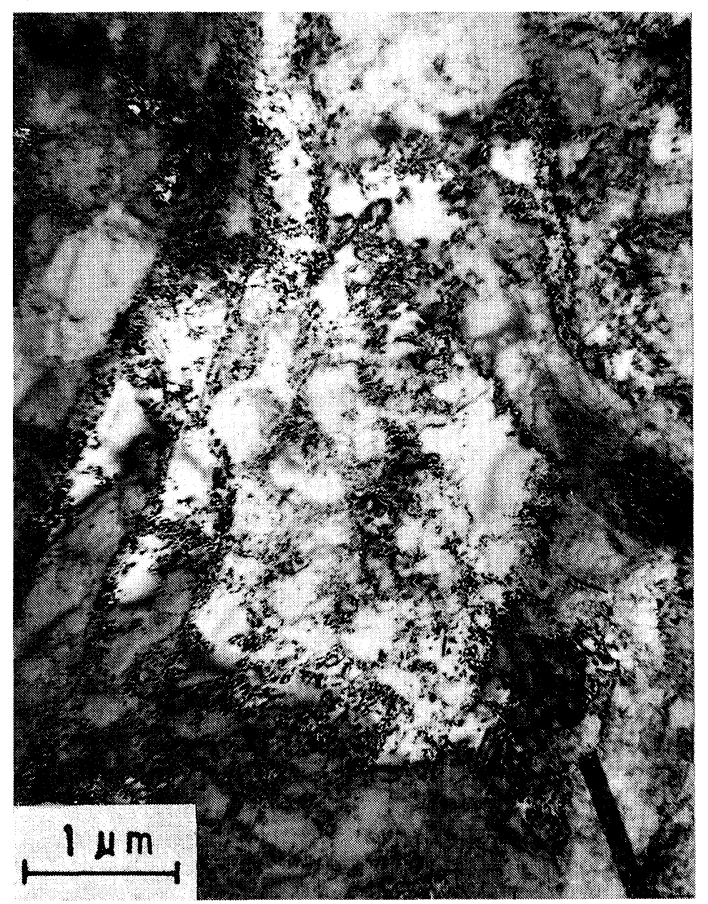

Figure 8 Transmission electron micrograph of $10 \%$ cold rolled $(\mathrm{CR}) \mathrm{HB}$ at $473 \mathrm{~K}$ : bright field (BF) image.

was also of $\langle 110\rangle \gamma$ orientation and the twin was found to orient itself along $\langle 112\rangle \gamma$ direction. The density of dislocations in this recovered grain is relatively small as compared to other grains where cell structures are the essential features. At very low degree of rolling (10\% reduction), plastic deformation is realized chiefly by multiple slip and besides, zones of uniform dislocations, there appear well shaped cell structures and a scattered mechanical twin band in one crystallographic plane (Figure 8).

At $30 \%$ reduction, profuse twinning in the form of planar bands can be found (Figure 9). The BF, dark field (DF) using (002) $)_{\mathrm{T}}$ reflection and SAD evidences shown in Figure, 9(a), (b) and (c) indicate that the planar bands shown in Figure 9(a) are mechanical twins (T) formed within suitably oriented grain of austenite $(\gamma)$. The indexing of the SAD pattern is shown in Figure 9(d). The habit plane of these twins is inclined to the electron beam (i.e. angle of specimen tilting $10^{\circ}$ ) resulting in the composite pattern $\langle 114\rangle \gamma /\langle 110\rangle_{\mathrm{T}}$. The measured volume fraction of the twins in suitably oriented grains is of the order of $35 \%$. In contrast, other grains exhibit well developed cell structures. Further rolling (i.e. $\leq 50 \%$ reduction) not only increases the amount of mechanical twins but also causes these twins to form in two intersecting crystallographic planes (Figure 10).

\section{DISCUSSION}

The examination of texture of $\mathrm{HB}$ exhibits that the $\mathrm{HB}$ is composed of grains whose orientations are concentrated along $\langle 110\rangle 60^{\circ} \mathrm{ND}$ fibre with $\{011\}\langle 112\rangle$ as major and $\{225\}\langle 554\rangle$ as minor components. In addition, recrystallised grains of cube orientation 

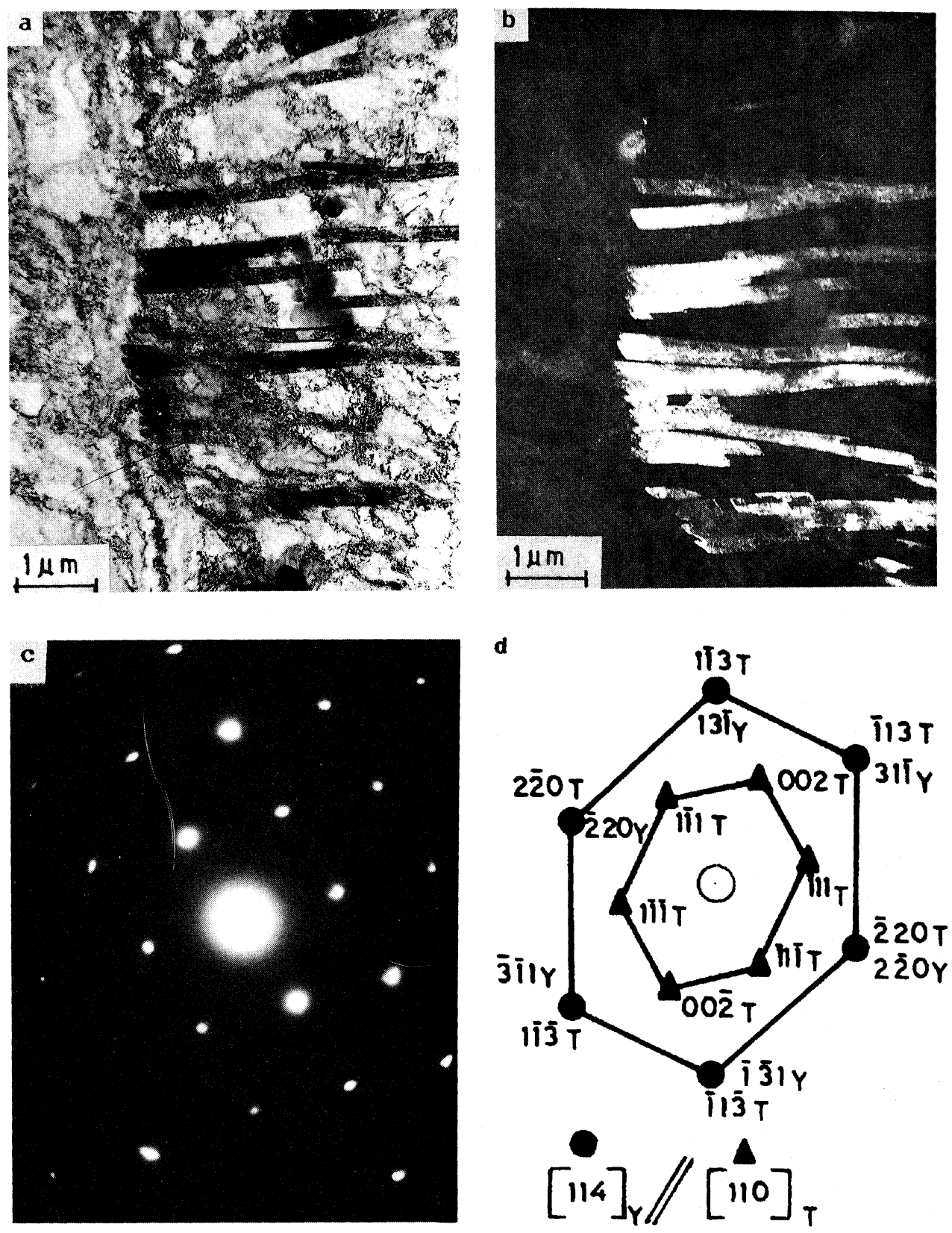

Figure 9 Transmission electron micrographs of $30 \%$ cold rolled (CR) HB at $473 \mathrm{~K}$ : a) bright field $(\mathrm{BF})$ image b) dark field (DF) image c) selected area diffraction (SAD) pattern d) key to SAD pattern. 


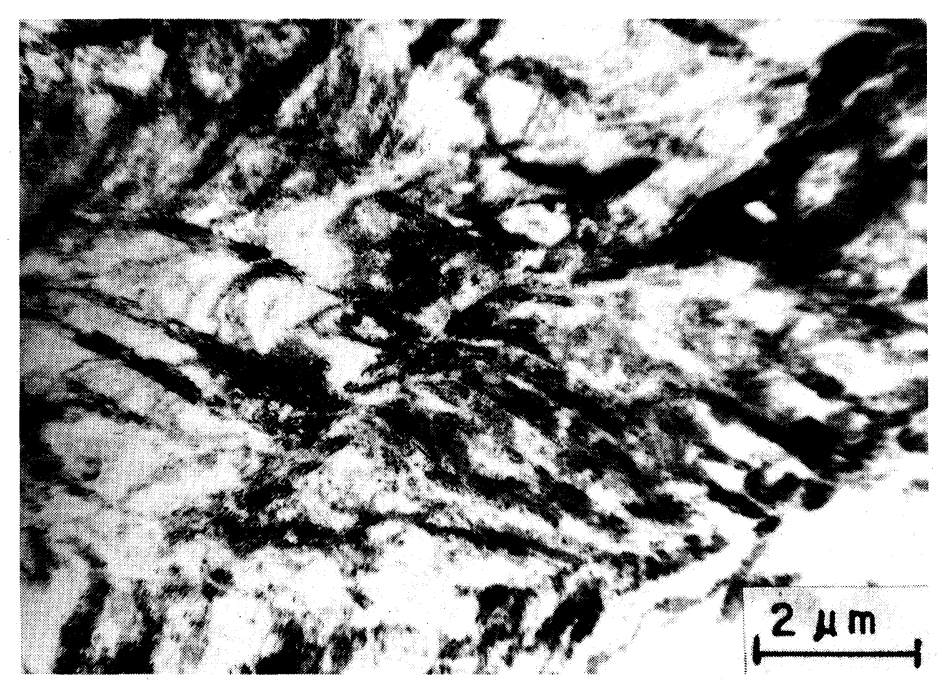

Figure 10 Transmission electron micrograph of 50\% cold rolled HB at $473 \mathrm{~K}$ : bright field image.

$\{001\}\langle 100\rangle$ and other RD rotated cubes are also present. The fibre $\langle 110\rangle 60^{\circ} \mathrm{ND}$ is one of the two fibres (i.e. $\langle 110\rangle / / \mathrm{ND}$ and $\langle 110\rangle 60^{\circ} \mathrm{ND}$ ) which develope in materials of high to medium SFE at low degrees of rolling. The minor orientation $\{225\}\langle 554\rangle$ is in between $\{112\}\langle 111\rangle$ and $\left\{\begin{array}{lll}4 & 4 & 11\end{array}\right\}\langle 11118\rangle$ and differs approximately $5^{\circ}$ from $\{112\}\langle 111\rangle$ and $3^{\circ}$ from $\left\{\begin{array}{lll}4 & 4 & 11\end{array}\right\}\left\langle\begin{array}{llll}11 & 11 & 8\end{array}\right\rangle$. Further, TEM features (Figure 7) indicate that the grain containing stacking faults and recovery twin is of $\{011\}\langle 112\rangle$ type - an indirect evidence - and is fully recovered.

At very low degrees of rolling (10\% CR), the presence of well developed cells and a scattered mechanical twin band as shown in Figure 8 indicates that dislocation glide by multiple slip predominates with a weak participation of twinning in one crystallographic plane. As the rolling is continued (i.e. at $30 \% \mathrm{CR}$ ), the orientation density at $\{011\}\langle 112\rangle$ and $\sim\{123\}\langle 634\rangle$ increases strongly and that at $\{225\}\langle 554\rangle$ and $\{011\}\langle 100\rangle$ increases moderately and also there is a considerable decrease in the density of cube orientation $\{001\}\langle 100\rangle$. This increase in orientation density at $\{011\}\langle 112\rangle$ and $\sim\{123\}\langle 634\rangle$ is partly due to starting texture and partly due to flow of cube $\{001\}\langle 100\rangle$ and other RD rotated cubes along $\langle 110\rangle / / \mathrm{ND}$ fibre and then along $\langle 110\rangle 60^{\circ} \mathrm{ND}$ fibre. Furthermore, orientations concentrate along $\langle 110\rangle / / \mathrm{ND}$ and $\langle 110\rangle 60^{\circ} \mathrm{ND}$ fibres (Figures 1,2 and 3). It is evident from Figure 4 that a maxima in orientation density at $\{225\}\langle 554\rangle$ occurs along with a scattering of $\{011\}\langle 100\rangle$ towards $\{144\}\langle 811\rangle$ and $\{332\}\langle 113\rangle$. On the other hand, the TEM evidence (Figure 9) clearly illustrate that the twinning is intensified and that profuse twinning occurs on one twinning system in suitably oriented grain (i.e. $\{225\}\langle 554\rangle$-an indirect evidence) but still multiple slip predominates which is menifested by the presence of well developed cells in other grains. The predominance of dislocation glide by slip in contrast to twinning is also corroborated by the plot of texture index with increasing degrees of rolling (Figure 5). It is worth to mention, here, that the rate of texture development (i.e. slope $=0.61$ ) is very close to that of coppertype (slope $=0.79$ ) calculated on the basis of relaxed Taylor model as extracted from Figure 1 (Leffers, 1993). On the contrary, the rate of texture development of brass- 
type calculated on the basis of modified Sachs model (Leffers, 1993) is 0.27 and even less. This small deviation of measured rate of texture development from the calculated copper-type is mainly due to the onset of twinning in suitably oriented grains. In addition, the very close agreement of the experimentally determined positions of $\langle 110\rangle 60^{\circ} \mathrm{ND}$ fibre with the theoretically calculated positions of $\langle 110\rangle 60^{\circ} \mathrm{ND}$ fibre (Figure 6$)$ further substantiates the dominant role of multiple slip. However, the shifting of measured positions of $\langle 110\rangle 60^{\circ} \mathrm{ND}$ fibre in the region of orientations between $\{123\}\langle 634\rangle$ and $\{225\}\langle 554\rangle$ from the calculated ones towards higher $\varnothing$ and lower $\emptyset_{1}$ values at $30 \%$ reduction again shows the onset of mechanical twinning. All these evidences \{i.e. i) a moderate to high increase in the orientation density of all texture components of the two fibres $\left(\langle 110\rangle / / \mathrm{ND}\right.$ and $\left.\langle 110\rangle 60^{\circ} \mathrm{ND}\right)$, ii) a very close agreement of the measured $\langle 110\rangle 60^{\circ} \mathrm{ND}$ fibre with the theoretically determined $\langle 110\rangle 60^{\circ} \mathrm{ND}$ fibre on the basis of FC Taylor model and, iii) near matching of the measured rate of texture development with the calculated rate of development of copper-type texture suggest that the initial development of rolling textures (at $30 \% \mathrm{CR}$ ) is similar to that of copper-type and that this development agrees fairly well with the predictions of FC Taylor model (Hirsch and Lücke, 1988c). Further, twinning is intensified with increasing degrees of rolling (30\% CR) and this marks the beginning of transition from copper-type texture to brasstype and is also responsible for the modest increase in the orientation density of $\{225\}\langle 554\rangle$. It follows from the observations (i.e. a modest increase in the orientation density of $\{225\}\langle 554\rangle$ and an apparant scattering of $\{011\}\langle 100\rangle$ towards $\{144\}\langle 811\rangle$ and $\{111\}\langle 112\rangle)$ that at low degrees of reduction (30\%CR) the increase of $\{225\}\langle 554\rangle$ peak due to multiple slip is in completion with simultaneous twinning and abnormal rotation.

At $50 \%$ rolling reduction, the first detectable decrease in the, otherwise, stable $\{225\}\langle 554\rangle$ orientation accompanied by an increase in orientation density of twin component $\{144\}\langle 811\rangle$ and also simultaneous formation of peak at $\{332\}\langle 113\rangle$ (Figure 4) show the dominant role of mechanical twinning (Wassermann, 1963) in intensifying the texture transition whereby the orientation $\{225\}\langle 554\rangle$ undergoes mechanical twinning to form $\{144\}\langle 811\rangle$ at $\emptyset_{1}, \emptyset, \emptyset_{2}=90,80$ and $45^{\circ}$ and also the simultaneous operation of abnormal rotation of the twin-parent bands towards $\{111\}\langle 112\rangle$. Furthermore, TEM evidence (Figure 10) in close agreement with the textural feature (Figure 4) reveal clearly that mechanical twinning in suitably oriented grains predominates. The twin orientation $\{144\}\langle 811\rangle$, instead of rotating towards $\{011\}\langle 100\rangle$ and then to $\{011\}\langle 112\rangle$ (Wassermann, 1963), rotates in the opposite direction towards $\{111\}\langle 112\rangle$ at $\emptyset=55^{\circ}$. This abnormal rotation has also been observed by other investigators (Asbeck and Mecking, 1978; Duggan et al., 1978) and indicates that slip occurs preferentially on $\{111\}$ plane common to the twin plane and finally stops at $\{332\}\langle 113\rangle$ at $\emptyset=65^{\circ}$. The formation of peak at $\{332\}\langle 113\rangle$ illustrates that the shear stress for abnormal slip has decreased considerably and approached zero value once the twin-parent lamellae (planar bands) become oriented with the rolling plane. It follows from texture evidence (Figure 4) and TEM feature (Figure 10) that mechanical twinning plays a vital role in texture transition from copper-type to brass-type as manifested by nearly equal density of the peaks at $\{332\}\langle 113\rangle$ and at $\{225\}\langle 554\rangle$ and also by the presence of equal volume fraction of twin and matrix bands in suitably oriented grains (i.e. $\{112\}\langle 111\rangle$ ) respectively. Further rolling (at $70 \% \mathrm{CR}$ ) causes the appearance of orientation $\{334\}\langle 110\rangle$ differing by $8^{\circ}$ from $\{111\}\langle 110\rangle$ by the onset of twinning of $\{123\}\langle 634\rangle$ followed by abnormal rotation of $\{123\}\langle 634\rangle$ and its twin $\{123\}\langle 634\rangle$ on $\{111\}$ plane parallel to the twin plane. Simultaneously with the operation of twinning 
and abnormal rotation, the formation of shear bands in the rotated twin-matrix regions leads to rotation of $\{332\}\langle 113\rangle$ and $\{111\}\langle 110\rangle$ to near $\{011\}\langle 100\rangle$ and $\{011\}\langle 112\rangle$ respectively as well as the re-establishment of multiple slip in the fine grains of shear bands at $90 \%$ CR causes the sharpening of final brass-type texture i.e. $\langle 110\rangle / / \mathrm{ND}$ fibre (Singh, Ramaswamy and Suryanarayana, 1992).

\section{CONCLUSIONS}

The statements made by Singh, Ramaswamy and Suryanarayana (1992) are correct and the Leffers's reinterpretations do not hold good. The above demonstrated facts pertain and lead to the following statements:

i) The initial texture development of brass-type is similar to that of copper-type at least upto $30 \%$ cold reduction and the texture consists of two limited fibres (i.e. , 110$\rangle / / \mathrm{ND}$ and $\left.\langle 110\rangle 60^{\circ} \mathrm{ND}\right)$.

ii) This texture development follows Taylor model (Full constraints).

iii) Mechanical twinning plays a dominant role in the transition of texture from coppertype to brass-type and twins $\{225\}\langle 554\rangle$ component to $\{144\}\langle 811\rangle$ in the form of twin - matrix bands (lamellae).

\section{Acknowledgements}

The author would like to express his gratitude to the management of RDCIS, SAIL, Ranchi for according the permission to publish this work.

\section{References}

1. Asbeck, H. O. and Mecking, H., (1978). Mat. Sci. Engg., 34, 111.

2. Blicharski, M. and Gorczyca S., (1978). Metal Sci., 12, 303.

3. Duggan, B. J., Hatherly, M., Hutchinson, W. B. and Wakefield, P. T. (1978). Metal Sci., 12, 343.

4. Hirsch, J. and Lücke, K. (1988a). Acta Metall., 36, 2863.

5. Hirsch, J. and Lücke, K. (1988c). Acta Metall., 36, 2883.

6. Hirsch, J., Lücke, K. and Hatherly, M. (1988b), Acta Metall., 36, 2905.

7. Hutchinson, W. B., Duggan, B. J. and Hatherly, M. (1979). Metals Tech., 6, 398.

8. Leffers, T. (1969). Textures in Research and Practice, Ed. J. Grewen and G. Wassermann, SpringerVerlag, Berlin, 120.

9. Leffers, T. (1993). Textures and Microstructures. (This Volume)

10. Leffers, T. and Blide - Sorensen, J. B. (1990). Acta Metall. Mater., 38, 1917.

11. Leffers, T. and Grum Jensen, A. (1968). Trans. Met. Soc. AIME, 242, 314.

12. Leffers, T. and Hansen, N. (1992). Modelling of Plastic Deformation and its Engineering Applications, Eds. S. I. Anderson et al., Ris $\varnothing$ National Laboratory, Roskilde, 57.

13. Leffers, T. and Juul Jensen, D. (1988). Textures and Microstructures, 8/9, 467.

14. Leffers, T. and Juul Jensen, D. (1991). Textures and Microstructures, 14-18, 933.

15. Pedersen, O. B. and Leffers, T. (1987). Constitutive Relations and Their Physical Basis, Eds. S. I. Anderson et al., Risø National Laboratory, Roskilde, 147.

16. Sachs, G. (1928). Z. Verein. Deut. Ing., 72. 734.

17. Singh, C. D., Ramaswamy, V. and Suryanarayana, C. (1991). Textures and Microstructures, 13, 227.

18. Singh, C. D., Ramaswamy, V. and Suryanarayana, C. (1992). Textures and Microstructures, 19, 101 .

19. Taylor, G. I. (1938). J. Inst. Metals, 62, 307.

20. Wassermann, G. (1963). Z. Metallk., 54, 61. 